\title{
Surveys on COVID-19 in nuclear medicine: what happened and what we learned
}

\author{
Salvatore Annunziata ${ }^{1}$. Domenico Albano ${ }^{2} \cdot$ Riccardo Laudicella $^{3} \cdot$ Matteo Bauckneht $^{4}$ on behalf of The Young \\ Committee of the Italian Association of Nuclear Medicine (AIMN)
}

Received: 1 September 2020 / Accepted: 12 September 2020 / Published online: 24 September 2020

(C) Italian Association of Nuclear Medicine and Molecular Imaging 2020

\begin{abstract}
Introduction To summarize and comment the available surveys on the impact of COVID-19 on Nuclear Medicine (NM) departments.

Methods Recent surveys about the impact of COVID-19 on Nuclear Medicine (NM) departments have been searched on available scientific database.

Results Three surveys have been found and commented. Some differences among the surveys should be kept in mind. All studies agreed that COVID-19 had a relevant impact on NM departments.

Conclusion Surveys about the impact of COVID-19 in NM could improve the departments' daily activities, to find a compromise between the security protocols suggested by international guidelines.
\end{abstract}

Keywords Survey · COVID · Nuclear Medicine

COronaVIrus Disease 19 (COVID-19) is still spreading worldwide, and the pandemic peak has probably not been reached yet. During the pandemic, Nuclear Medicine (NM) departments have been involved in several changes and issues, such as new scheduling praxes for NM diagnostic and therapeutic procedures, the assistance of patients with diagnosed or suspected COVID-19, and the prevention of COVID-19 spreading in the departments. For these reasons, some research groups promoted different international surveys to understand the impact of the pandemic on NM departments [1-3].

The Young Group of the Italian Association of Nuclear Medicine (AIMN) developed the first international survey

Salvatore Annunziata

salvatoreannunziata@live.it

1 Nuclear Medicine Unit, IRCSS Regina Elena National Cancer Institute, Via Elio Chianesi 53, 00144 Rome, Italy

2 Department of Nuclear Medicine, University of Brescia and Spedali Civili Brescia, Brescia, Italy

3 Nuclear Medicine Unit, Department of Biomedical and Dental Sciences and of Morpho-Functional Imaging, University of Messina, Messina, Italy

4 Nuclear Medicine Unit, IRCCS Ospedale Policlinico San Martino, Genoa, Italy to evaluate the impact of COVID-19 in NM and performed a preliminary report of the obtained responses [1]. Twohundred and ninety-six individual responders from 220 departments were evaluated. Most of the responders were from Europe (67\%). Approximately, all departments changed their scheduling praxes due to the pandemic (97\%). In most departments, scheduled diagnostic and therapeutic procedures were allowed but quantitatively reduced (51\%). A significant reduction (more than 20\%) of diagnostic and therapeutic procedures affected $90 \%$ and $72 \%$ of the departments, respectively. Incidental COVID-19 signs in NM exams occurred in about half of the departments. Only a few departments were closed or shifted to assist patients with COVID-19 (16\%). Finally, most of the responders thought that the pandemic would not permanently change the work of NM departments in the future (64\%) [1].

Another international research group promoted a survey about the global impact of COVID-19 on NM departments [2]. A total of 434 responses from 72 countries were registered and analysed. The authors reported an average decline of $54 \%$ in diagnostic procedures. Namely, PET/CT scans decreased by an average of $36 \%$, sentinel lymph-node procedures decreased by $45 \%$, lung scans by $56 \%$, bone scans by $60 \%$, myocardial studies by $66 \%$, and thyroid studies by $67 \%$. About radionuclide therapies, they reported a reduction 
Table 1 Comparison of the mains results emerging by the three recent surveys performed about the impact of COVID-19 pandemic on NM departments in different countries worldwide

\begin{tabular}{lllll}
\hline Authors, [ref] & Journal name & \multicolumn{3}{l}{ Reduction of NM procedures (\%) } \\
\cline { 3 - 5 } \cline { 3 - 4 } & & $\begin{array}{l}\text { Conventional } \\
\text { scintigraphy }\end{array}$ & PET & Therapy \\
\hline Annunziata et al. [1] & Eur J Nucl Med Mol Imaging & $70^{\mathrm{a}}$ & $11^{\mathrm{a}}$ & $19^{\mathrm{a}}$ \\
Freudenberg et al. [2] & J Nucl Med & $54^{\mathrm{b}}$ & $36^{\mathrm{b}}$ & $45^{\mathrm{b}}$ \\
Freudenberg et al. [3] & Nuklearmedizine & $40^{\mathrm{b}}$ & $14^{\mathrm{b}}$ & $30^{\mathrm{b}}$ \\
\hline
\end{tabular}

NM nuclear medicine, COVID-19 COronaVIrus Disease 19

${ }^{a}$ Worst reduction over the total of the responders

${ }^{\mathrm{b}}$ Mean reduction among the responders of $45 \%$, ranging from over $76 \%$ in Latin America and Asiatic South-East to $16 \%$ in South Korea and Singapore. Survey results showed that $52 \%$ of the participating sites limited their $99 \mathrm{mTc} / 99 \mathrm{Mo}$ generator purchases, and $12 \%$ of them temporarily canceled orders. Insufficient supplies of essential materials (radioisotopes, generators, and kits), especially for $99 \mathrm{mTc} / 99 \mathrm{Mo}$ generators and $131 \mathrm{I}$, were mainly reported in Africa, Asia, and Latin America [2].

The same research group promoted a regional analysis of the impact of COVID-19 on NM in Germany, Austria, and Switzerland [3]. In this survey, 113 complete responses were recorded. Nearly, all participants reported a decline of NM diagnostic procedures. The mean reduction for PET/CT, scintigraphy of bone, myocardium, lung, thyroid, sentinel lymph-node was of $14 \%, 47 \%, 48 \%, 41 \%, 58 \%$, and $25 \%$, respectively. Furthermore, $76 \%$ of the participants reported a reduction in therapeutic procedures, mainly for benign thyroid disease (42\%) and radiosynoviorthesis (54\%), while tumor therapies remained almost stable. Also, half of the participants reported a shortage of staff's protective equipment [3]. A summary of the results from the three surveys is reported in Table 1.

Some limitations and differences among these three surveys should be kept in mind: the structure of the questionnaires, the methods used to invite the participants, the number of responses from the same department, and the discrepancies due to geographical criteria and pandemic stages. Nevertheless, all the three studies agreed that COVID-19 had a relevant impact on NM departments in the scheduling praxes for diagnostic and therapeutic procedures, the assistance of patients with diagnosed or suspected COVID19 , and the prevention of COVID-19 spreading in the departments.

The decrease and/or the postponement of medical procedures underlined that potentially many patients have been "missed" during the pandemic, and this may impact on the disease's clinical course, potentially affecting also the quality of life and survival, opening an ethical debate. Namely, according to the global survey by Freudenberg et al. [2], it is unknown whether the decrease in the NM procedures is attributable to patients' fears and preferences, safety precautions adopted by NM centers, disruption of supply chains and logistical challenges, or a combination of all the above [2].

Nevertheless, some suggestions for future actions could be derived from all these studies as: awareness of the main guidelines for the organization of the departments; scheduling of NM examinations considering urgency and appropriateness criteria; robust screening process as phone or on-site pre-triage; attention to simply rules (also hygienic), as proper distancing and escalation for suspected cases; further investigations for possible indications of $\mathrm{NM}$ examinations in the ordinary management of COVID-19 [1].

According to Freudenberg et al. [2], it is our responsibility to continue providing essential services to ascertain adequate diagnostic and therapeutic patient services, while ensuring proper hygienic measures, thus safeguarding the health of staff, patients and the public. It is also essential to address the significant disruptive economic impact of this pandemic on healthcare systems, with particular interest to NM departments. The more we know about the current and upcoming challenges, the better we can learn and adapt collectively to them [2]. For instance, the fruitful cooperation between different NM departments to provide a prompt radionuclide treatment to patients coming from different Italian regions should be underlined.

The lesson we learned from this global experience should be useful to improve NM departments' daily activities, to find a compromise between the security protocols suggested by international guidelines and the need to continue medical assistance in favor of the emergency needs without sacrificing patients' healthcare.

\section{Compliance with ethical standards}

Conflict of interest The authors declare no conflict of interest.

Research involving human participants and/or animals The present manuscript does not involve human participants. 
Informed consent The present manuscript does not require an informed consent collection.

\section{References}

1. Annunziata S, Bauckneht M, Albano D et al (2020) Impact of the COVID-19 pandemic in nuclear medicine departments: preliminary report of the first international survey. Eur J Nucl Med Mol Imaging 47(9):2090-2099

2. Freudenberg LS, Paez D, Giammarile F et al (2020)Global impact of COVID-19 on nuclear medicine departments: an international survey in April 2020. J Nucl Med (published online ahead of print, 24 July 2020)

3. Freudenberg LS, Dittmer U, Herrmann K (2020) Impact of COVID-19 on nuclear medicine in Germany, Austria and Switzerland: an international survey in April 2020. Auswirkungen von COVID-19 auf die Nuklearmedizin in Deutschland, Österreich und der Schweiz: Eine internationale Umfrage im April 2020. Nuklearmedizin 59(4):294-299

Publisher's Note Springer Nature remains neutral with regard to jurisdictional claims in published maps and institutional affiliations. 